\title{
Re-examination of the Observed Decadal Variability of Earth Radiation Budget using Altitude-corrected ERBE/ERBS Nonscanner WFOV Data
}

\author{
Takmeng Wong ${ }^{*}$, Bruce A. Wielicki, and Robert B. Lee, III \\ NASA Langley Research Center, Hampton, VA \\ G. Louis Smith \\ National Institute of Aerospace, Hampton, VA \\ Kathryn A. Bush \\ Science Applications International Corporation, Hampton, VA
}

Submitted as a Letter to the Journal of Climate

June 2005

*Corresponding author address: MS 420, NASA Langley Research Center, Hampton, VA, 23681-2199; email: takmeng.wong@ nasa.gov 


\begin{abstract}
$\underline{\text { Abstract }}$
This paper gives an update on the observed decadal variability of Earth Radiation Budget using the latest altitude-corrected Earth Radiation Budget Experiment (ERBE)/Earth Radiation Budget Satellite (ERBS) Nonscanner Wide Field of View (WFOV) instrument Edition3 dataset. The effects of the altitude correction are to modify the original reported decadal changes in tropical mean (20N to $20 \mathrm{~S}$ ) longwave (LW), shortwave (SW), and net radiation between the 1980s and the 1990s from 3.1/-2.4/-0.7 to 1.6/-3.0/1.4 $\mathrm{Wm}^{-2}$ respectively. In addition, a small SW instrument drift over the 15-year period was discovered during the validation of the WFOV Edition3 dataset. A correction was developed and applied to the Edition3 dataset at the data user level to produce the WFOV Edition3_Rev1 dataset. With this final correction, the ERBS Nonscanner observed decadal changes in tropical mean LW, SW, and net radiation between the 1980s and the 1990s now stand at $0.7 /-2.1 / 1.4 \mathrm{Wm}^{-2}$, respectively, which are similar to the observed decadal changes in the HIRS Pathfinder OLR and the ISCCP FD record; but disagree with the AVHRR Pathfinder ERB record. Furthermore, the observed interannual variability of near-global ERBS WFOV Edition3_Rev1 net radiation is found to be remarkably consistent with the latest ocean heat storage record for the overlapping time period of 1993 to 1999 . Both data sets show variations of roughly $1.5 \mathrm{Wm}^{-2}$ in planetary net heat balance during the 1990s.
\end{abstract}




\section{Introduction}

Continuous monitoring of the Earth radiation budget (ERB) at the top of atmosphere (TOA) is essential for understanding climate and climate variability on Earth. Wielicki et al. (2002a) reported large decadal changes in tropical mean (20N to 20S) Earth radiation budget between the 1980s and the 1990s based on the longest running single ERB time series produced from the Earth Radiation Budget Experiment (ERBE; Barkstrom, 1984) Earth Radiation Budget Satellite (ERBS) Nonscanner Wide Field of View (WFOV) instrument Edition2 data record. The results also were based on a range of overlapping scanning and nonscanning ERB instruments; including Nimbus-7 Nonscanner (Bess et al., 1989), ERBE/ERBS Scanner, the Scanner for Radiation Budget (ScaRaB) instrument (Kandel et al., 1998) on the Meteor satellite, the ScaRaB Scanner (Duvel et al., 2001) on the Resurs Satellite, the Clouds and the Earth's Radiant Energy System (CERES, Wielicki et al., 1996) Scanner on the TRMM satellite and the CERES Scanner on the Terra Satellite.

Since that report, a complete re-examination of the ERBE/ERBS Nonscanner WFOV dataset, prompted by new findings on the possible effect of small ERBS altitude changes over the 15-year period, has been completed. This satellite altitude change and its effects on the top of the atmosphere ERB are discussed in section 2 using the new altitude-corrected WFOV Edition3 dataset. Section 3 presents results on a small WFOV shortwave instrument drift and its effects on the ERB record. This instrument drift was discovered during the validation of the WFOV Edition3 dataset and is not currently included in the archived WFOV Edition3 data, but a simple correction method is available to the data user to remove this instrument artifact. Sections 4 and 5 compare the 
latest ERBS Nonscanner WFOV record with existing satellite-based time series of ERB from the High Resolution Infrared Radiation Sounder (HIRS) Pathfinder Outgoing longwave radiation (OLR, Mehta and Susskind, 1999) dataset, the International Satellite Cloud Climatology Project (ISCCP) FD dataset (Zhang et al., 2004), and the Advanced Very High Resolution Radiometer (AVHRR) Pathfinder ERB dataset (Stowe et. al., 2002), as well as records from the most recent ocean heat storage dataset based on both ocean in-situ vertical soundings and satellite altimeter measurements (Willis et al. 2004). Summary and conclusions are given in Section 6.

\section{Altitude-corrected WFOV Edition3 data}

ERBS altitude (shown in Figure 1) slowly dropped from $611 \mathrm{~km}$ to $585 \mathrm{~km}$ over the first 15 years of the mission. The changes in satellite altitude can directly affect the ERBS Nonscanner WFOV TOA fluxes through the ERBS Nonscanner inversion process in which satellite altitude observed fluxes are converted to TOA fluxes (Lee et al., 2003). Unlike the scanner instrument, which sees only small portions of the Earth surface, the Nonscanner WFOV instrument field-of-view at satellite altitude contains the entire earth disk and the surrounding deep space. The amount of energy received at the Nonscanner WFOV instrument is therefore inversely proportional to the square of the distance between the instrument and the Earth's center. As the altitude dropped over the 15-year period, the Nonscanner WFOV instrument recorded a small steady increase in satellite altitude fluxes. The ERBS WFOV Edition2 data analysis system was designed to account for changes in satellite altitude, but the original algorithm designed this function to provide a correction in the case of a non-circular satellite orbit. It was recently discovered 
that the altitude correction was disabled in the case of a near-circular orbit, and therefore did not apply to correct the average spacecraft altitude change that occurred later in the extended ERBS mission. The main effect of this altitude change is a small increase $(\sim 0.6 \%)$ in both TOA outgoing longwave radiation (LW) and reflected shortwave radiation (SW) over the 15-year period.

Based on this new information, the entire ERBE/ERBS Nonscanner WFOV Edition2 data record was reprocessed to Edition3 data using a set of time dependent correction coefficients derived from time series of the ERBS altitude record. Figure 2 shows the time series of 36-day averaged tropical mean (20N to 20S) ERBS Nonscanner WFOV radiative energy budget with (Edition3, solid lines) and without (Edition2, dashed lines) this satellite altitude correction. The 36-day mean is used to reduce diurnal aliasing in the Nonscanner dataset resulting from a shift in the local time of satellite observations over the 15-year period (Wielicki et al., 2002b). Figure 2 is in the form of deseasonalized anomalies with respect to the 1985 to 1989 climatology. This was done because the seasonal cycles in LW, SW, and net flux are large enough to make decadal variability difficult to visualize. The effect of the altitude correction is to reduce the magnitude of the tropical LW flux change from the 1980s to 1990 s from the original 3.1 to $1.6 \mathrm{Wm}^{-2}$. The correction increases the magnitude of the SW flux decadal change from -2.4 to -3.0 $\mathrm{Wm}^{-2}$. The correction also increases the net radiation decadal change from $-0.7 \mathrm{Wm}^{-2}$ (cooling of the tropics) to $1.4 \mathrm{Wm}^{-2}$ (heating of the tropics). 


\section{WFOV Shortwave Instrument Drift}

In addition to the satellite altitude decrease over the 15-year period, the ERBS WFOV shortwave instrument also appears to have a residual instrument trend that is not fully corrected by using the bi-weekly solar constant calibrations. This shortwave instrument trend, on the level of $1 \%$ over the 15 -year period, was discovered during the validation of the ERBE/ERBS WFOV Edition3 dataset. The mostly likely cause of this shortwave trend, based on the most recent instrument engineering study, is due to nonuniform exposure of the Nonscanner WFOV shortwave sensor dome to UV radiation during spacecraft sunrise and sunset over the 15-year period. In particular, the sides of the SW filter dome on the Nonscanner WFOV instrument receive more UV exposure than the top of the dome. This can lead to a slight difference in the dome transmission between viewing a small angle of view source like the sun, versus a wide angle of view target like the Earth. Since the Nonscanner WFOV instrument does not carry a dedicated longwave sensor, the longwave fluxes during daytime are determined from the total channel ( 0.2 to 100 micron) minus the shortwave channel ( 0.2 to 5 micron). Thus, this residual shortwave instrument trend can also directly affect the reported daytime longwave fluxes. During nighttime, the longwave fluxes are determined directly from the total channel since there are no shortwave measurements at night.

Figure 3 shows the time series of daytime longwave flux (red curve), nighttime longwave flux (blue curve) and day-minus-night longwave flux difference (green curve) over the tropics from the ERBS Nonscanner WFOV Edition3 data record over the 15year period. These time series are deduced directly from instantaneous grid box data and contain slightly more sampling noise than the traditional monthly mean data. 
Nevertheless, the figure clearly shows that the day-minus-night longwave difference is slightly increasing over time. This results from the fact that the nighttime longwave shows no change, but the daytime longwave has increased slightly over the same period. Figure 3 suggests that the WFOV total channel is stable over time (consistent with solar constant checks), but the WFOV shortwave channel has drifted slightly over the same period. Basically, the shortwave dome has degraded slightly over time and allows less solar energy onto the WFOV shortwave detector, thus artificially producing a lower shortwave flux and a higher longwave flux during daytime.

The ERBS Nonscanner measurements discussed up to this point are based on data from the Wide Field of View (WFOV) instruments. The ERBS Nonscanner instrument package also contains a set of Medium Field of View (MFOV) instruments that record the Earth reflected solar and total spectrum energy with a field of view of about $500 \mathrm{~km}$ diameter. These MFOV instruments are adjacent to the WFOV instruments on the ERBS spacecraft. Unlike the WFOV instruments, the MFOV sensors are completely shielded from the Sun during Earth viewing operation. Therefore the MFOV data should show no trend in the day minus night longwave flux over this period. This is evident in Figure 4, which shows the MFOV daytime longwave flux, nighttime longwave flux and day minus night longwave flux difference time series over the ERBS 15-year period. The day minus night MFOV longwave flux differences, the daytime longwave flux, and nighttime longwave flux are very stable over this time period. The short time scale noise of these fluxes, however, is much larger than the WFOV, since the MFOV covers the daytime tropics only once every 4 days. The MFOV data supports the hypothesis that non-uniform 
solar exposure on the Nonscanner WFOV SW dome has caused the observed drift in the Nonscanner WFOV measurement over the 15-year period.

A correction for this shortwave sensor trend is derived from the WFOV Edition3 dataset using a simple linear regression technique and applied to the WFOV Edition3 time series shown in Figure 2. This shortwave sensor drift corrected data will now be referred to as WFOV Edition3_Rev1 data. Note that the WFOV SW sensor trend correction is not currently included in the archived WFOV Edition3 dataset. However, this correction is available to data users through the online WFOV Edition3 data quality summary so they can apply the correction directly to the WFOV Edition3 data in future studies.

In general, the shortwave sensor drift correction further reduces the magnitude of the tropical mean decadal changes in LW flux from $1.6 \mathrm{Wm}^{-2}$ to about $0.7 \mathrm{Wm}^{-2}$, and reduces the magnitude of the tropical mean decadal changes in SW flux from $-3.0 \mathrm{Wm}^{-2}$ to about $-2.1 \mathrm{Wm}^{-2}$ between the 1980s and the 1990s. Note that the change in WFOV SW channel has no effect on net flux trends, which remain at $1.4 \mathrm{Wm}^{-2}$. Net fluxes depend only on the WFOV total channel, which has no dome degradation, and has shown consistency in solar calibration to $0.1 \%$ over the first 15 years of the ERBS mission (Lee et al., 2002).

Figure 5 shows an updated comparison of the new ERBS Nonscanner WFOV Edition3_Rev1 deseasonalized tropical mean (20N to 20S) flux anomalies time series to climate models. The comparison between Nonscanner WFOV Edition3_Rev1 deseasonalized LW anomalies and climate models is now in much better agreement, with the exception of the $1998 \mathrm{El}$ Nino anomaly peak, but sizeable differences in 
deseasonalized SW flux and net flux anomalies remain. Note that the 1991 to $1993 \mathrm{Mt}$. Pinatubo aerosol signal was not provided to the climate models for the simulations.

\section{Comparison with other satellite-based decadal Earth radiation budget records}

Several satellite-based decadal Earth radiation budget datasets of varying quality are available to the public. This section compares the data sets. Figure 6 shows a comparison of the ERBE/ERBS Nonscanner WFOV Edition3_Rev1 deseasonalized tropical mean $(20 \mathrm{~N}$ to $20 \mathrm{~S})$ radiative anomaly record (anomalies with respect to the 1985 to 1989 climatology) against deseasonalized anomalies from HIRS Pathfinder OLR data, AVHRR Pathfinder ERB data, and ISCCP FD data. For the LW component of the ERB, the four different datasets are consistent with each other during the first half of the data record. During the second half of the data record, the ERBS Nonscanner LW, HIRS Pathfinder OLR, and the ISCCP FD LW are in close agreement. The AVHRR Pathfinder LW, however, shows much lower values than the other time series during the later part of this data record. These problems are the result of instrument intercalibration and satellite orbit changes (time of day sampling) as discussed in Jacobowitz et al. (2003).

For the SW component of the ERB, the ERBS Nonscanner WFOV Edition3_Rev1 and the ISCCP FD data again agree well with each other over the entire span of the data period. HIRS Pathfinder provides only LW fluxes, so no SW or net flux comparisons are possible. The AVHRR Pathfinder SW data do not agree well with the two other datasets. The AVHRR data contain large shifts throughout the time series, again consistent with issues of intercalibration and changes in satellite orbit diurnal sampling. 
The net component of the ERB is the combined effect of both LW and SW fluxes. While both the ERBS Nonscanner WFOV Edition3_Rev1 data and the ISCCP FD data are very similar to each other, the AVHRR Pathfinder net data again diverge from the other two datasets. Table 1 further summarizes the results of decadal changes in Earth radiation budget between the 1980s and the 1990s for these four datasets and the ERBS Nonscanner WFOV Edition2 and Edition3 datasets. In general, there is good agreement among ERBS Nonscanner WFOV Edition3_Rev1, HIRS Pathfinder OLR and ISCCP FD data record. All three datasets show similar decadal changes in ERB with the same sign and similar magnitude. The AVHRR Pathfinder ERB dataset, however, disagrees with the other three datasets in both sign and magnitude. Jacobowitz et al. (2003) showed a promising technique for reducing these data problems in the current AVHRR Pathfinder ERB dataset. This corrected AVHRR Pathfinder dataset, however, is still under development and is not yet available to the public. An examination of Figure 4 from Jacobowitz et al. (2003) indicates that the comparable numbers for Table 1 would be about $-2 \mathrm{Wm}^{-2}$ for LW flux, $+2 \mathrm{Wm}^{-2}$ for $\mathrm{SW}$ flux, and near 0 for net flux. But intersatellite shifts of 4 to $5 \mathrm{Wm}^{-2}$ occur even in the corrected AVHRR Pathfinder data from Jacobowitz et al. (2003). We conclude that both the AVHRR pathfinder and the corrected AVHRR Pathfinder ERB datasets are not sufficiently accurate to resolve decadal changes in tropical mean or global scale radiative fluxes. They may be more useful for regional climate signals, but these are not compared here. 


\section{Comparison with ocean heat storage data}

Willis et al. (2004) provides new estimates of annual ocean heat storage for 1992 to 2002 using a combination of improved in-situ temperature profile sampling and constraints on thermal expansion from satellite global ocean altimeter observations. The major advantage of this dataset over previous ocean estimates is the use of global altimeter data to supplement sparse in-situ sampling in the southern hemisphere oceans.

On a global annual scale, net radiation and ocean heat storage should be in phase and of the same magnitude. All other forms of heat storage in the Earth system are factors of 10 or more smaller than ocean heat storage (Levitus, 2001). Previous ocean heat storage data sets required 5 to 10 year averages to reduce sampling errors. The Willis et al. (2004) analysis demonstrated a sampling error of $0.4 \mathrm{Wm}^{-2}$ for global annual ocean heat storage.

Figure 7 shows a direct comparison of these new ocean heat storage anomalies against 12-month running mean ERBE/ERBS Nonscanner WFOV Edition3_Rev1 and CERES/Terra Scanner ES4 Edition2_Rev1 net flux anomalies. The CERES/Terra Scanner results are global and the ERBE/ERBS Nonscanner WFOV results cover $60 \mathrm{~N}$ to $60 \mathrm{~S}$ (or $\sim 90 \%$ of the Earth surface). While spatial sampling error is the dominant source of uncertainty in the ocean data, absolute calibration uncertainties dominate the radiation budget data. For a comparison of interannual variations, however, we can remove the mean calibration uncertainty by requiring agreement for the average of all overlapping data for each instrument time series (e.g. 1984 to 1999 average for ERBS). Note that Willis et al. (2004) estimate the 10 year average uncertainty in ocean heat storage from 1992 to 2002 as $\sim 0.2 \mathrm{Wm}^{-2}$. The interannual net flux anomalies in Figure 7 from the 
ERBS Nonscanner WFOV and CERES Scanner agree to within the ocean heat storage sampling uncertainties, with 1-sigma difference in the anomalies of $0.4 \mathrm{Wm}^{-2}$. The two times series are in phase with each other, consistent with the constraint of planetary energy balance.

This is a remarkable result given the totally independent physical measurement and sampling of the ocean heat storage data and the ERB datasets. The net flux anomalies within a single decade can be as large as $1.5 \mathrm{Wm}^{-2}$ according to both the ERB and the ocean storage data. The data agree that the ERBS Nonscanner WFOV net radiation anomalies shown in Figure 5 are accurate to better than $0.5 \mathrm{Wm}^{-2}$. The large $1.5 \mathrm{Wm}^{-2}$ change is most likely dominated by changes in cloudiness since aerosol radiative forcing estimates for this period show no large changes beyond the 1991-1993 Mt. Pinatubo cooling.

\section{Summary and Conclusions}

The original and Edition2 ERBE/ERBS Nonscanner WFOV data contain small systematic errors that can affect the interpretation of decadal changes. Specifically, ERBS altitude slowly dropped from $611 \mathrm{~km}$ to $585 \mathrm{~km}$ over the 15 -year period. This introduces a $0.6 \%$ correction to the decadal changes reported in a previous study. This altitude correction has been used to produce an updated ERBS Nonscanner WFOV Edition3 dataset.

The ERBS Nonscanner WFOV SW sensor dome transmission corrections determined by bi-weekly solar constant observations appear to have underestimated the change by about $1 \%$ over the first 15 years of the mission. The $1 \%$ correction to the SW 
sensor is not currently incorporated into the archived WFOV Edition3 dataset and can result in an additional $1 \mathrm{Wm}^{-2}$ correction to the decadal changes in both $\mathrm{LW}$ and $\mathrm{SW}$ fluxes. The drift correction, however, is available to data users through the WFOV Edition3 data quality summary so they can apply the correction to the WFOV Edition3 data and make them into WFOV Edition3_Rev1 data. Overall, the combined effects of altitude correction and SW sensor drift correction change the values of the reported decadal changes in tropical mean (20N to $20 \mathrm{~S})$ Earth radiation budget in TOA LW/SW/net radiation between the $1980 \mathrm{~s}$ and the 1990 s from $3.1 /-2.4 /-0.7 \mathrm{Wm}^{-2}$ to $0.7 /$ 2.1/1.4 $\mathrm{Wm}^{-2}$, respectively.

Comparison of decadal changes in ERB with existing satellite-based decadal radiation datasets shows very good agreement among ERBS Nonscanner WFOV Edition3_Rev1, HIRS Pathfinder OLR, and ISCCP FD datasets. The AVHRR Pathfinder ERB dataset, however, does not compare well against the ERBS Nonscanner WFOV and the two other ERB datasets, either in the normal AVHRR Pathfinder data, or in the corrected AVHRR Pathfinder data. Discontinuities in the AVHRR data remain too large for detection of the climate changes shown in the other data sets.

Comparison of interannual variability of net flux anomalies between ocean heat storage data and the ERB datasets shows remarkable agreements in both phase and magnitude of these two very different datasets. The ocean heat storage data agree with the level of interannual variability found in the radiation data. This variation is larger than known variations in aerosol or other radiative forcings in the late 1990s, and suggests a closely linked variation in global ocean heat storage and global cloud net radiative forcing. Because phase lag is not expected between these two variables, it remains 
unclear if slight changes in ocean surface temperature and surface heat fluxes are changing clouds, or if clouds are changing ocean heat storage.

\subsection{Discussions}

The magnitude of the global ocean heat storage and net radiation changes have several implications for understanding climate change.

1. The new results do not support the recent Iris hypothesis (Lindzen et al. 2001, Lin et al. 2004). As tropical and global SST warms in the late 1990s during the 97/98 El Nino, the Iris negative feedback predicts net flux to decrease (ocean cooling) as opposed to the increase (ocean heating) seen in Figure 7.

2. The ocean heat storage and net radiation data, while showing relatively large interannual variability, are consistent with heating predicted from current state of the art coupled ocean/atmosphere climate models. The anticipated change in anthropogenic radiative forcing over the next few decades is estimated as $\sim 0.6 \mathrm{Wm}^{-2}$ per decade (IPCC, 2001). The interannual variability in net radiation is of similar magnitude $\left(+/-0.7 \mathrm{Wm}^{-2}\right)$. Note that the ocean heat storage dataset for single annual mean values has a sampling uncertainty of $0.4 \mathrm{Wm}^{-2}(1 \sigma)$ so that the larger range of variation in ocean heat storage is more likely due to its larger sampling noise. The radiation data set has a larger mean bias uncertainty (absolute calibration) but smaller sampling error than the ocean heat storage data. The 10-year average of ocean heat storage is about $0.6 \mathrm{Wm}^{-2}$, similar to the levels predicted by current climate models for anthropogenic global warming scenarios (IPCC 2001, Hansen et al. 2005). 
3. The net radiation and ocean heat storage variability predict that studies of cloud feedback in the climate system will require extremely accurate long time series of both ocean heat storage data as well as clear-sky, all-sky and cloud radiative forcing observations. With anticipated anthropogenic radiative forcing changes of $0.6 \mathrm{Wm}^{-2}$ per decade, cloud radiative forcing changes of only $0.3 \mathrm{Wm}^{-2}$ per decade can represent $50 \%$ changes in climate sensitivity. Both ocean heat storage and radiation data sets will require intense examination to verify these subtle but critical changes. The GEWEX Radiation Panel is currently beginning a Radiative Flux Assessment of both TOA and surface radiative fluxes consistent with this need. Independent data sets and high accuracy and stability will be critical. Our results support the need for a 21st climate observing principle: independent observations, with independent analysis of each climate data set.

4. The data suggest that a key test of coupled climate models will be the observed interannual variations in ocean heat storage and net cloud radiative forcing. It will be necessary to unscramble these two very different physical processes in a cause and effect linkage on decadal time scales. On the basis of purely time scale, one hypothesis would suggest that the long time scale ocean dynamics variability drives the very short time scale cloud processes. But the ocean heat storage is dominated not by surface temperature change, but by changes down to depths of $1000 \mathrm{~m}$ (Levitus, 2001; Willis et al., 2004). Near surface SST changes are not constrained on the decadal time scale to simply follow changes in ocean heat storage. Therefore, the physical link of ocean heat storage to drive cloudiness changes is not clear. The alternative hypothesis is that changes in cloudiness are driving changes in ocean heat 
storage. In this scenario the cloudiness changes are the result of either systematic anthropogenic climate change (e.g. changing equator to pole temperature gradient) and/or natural variability. The ocean heating is then a response to changes in net cloud radiative forcing that modifies the surface ocean energy balance. The fully coupled system is much more complicated than described, and the examples are only meant to highlight the type of challenges ahead.

5. The ERBS Nonscanner WFOV data for November 1999 through current should be reprocessed to account for the 15-degree shift from the normal nadir pointing of these instruments on the ERBS spacecraft. The data will provide a key independent time series in the CERES time frame, and will cover the current gap in the ERB record from October 1999 through February 2000.

6. The intercomparison of the corrected ERBS Nonscanner WFOV with other radiation data sets reaffirms the critical need for overlapped and continuous climate data records. Figure 8 shows the new version of Fig.1 from Wielicki et al. (2002a) with the new ERBS WFOV Edition3_Rev1 data. The Scanner and Nonscanner records no longer agree as well as before. The disagreement, however, is within the absolute accuracy of the instruments for calibration of SW and LW fluxes: $2 \mathrm{Wm}^{-2}$ for ERBE and ScaRaB and $1 \mathrm{Wm}^{-2}$ for CERES. As a result, for non-overlapped climate records, differences of up to $3 \mathrm{Wm}^{-2}$ are within the absolute calibration uncertainty. This is analogous to the same issue in solar constant measurements and most climate measurements. Even the most accurately calibrated instruments are typically not sufficient to handle gaps in the data record. 
Acknowledgments: The authors would like to thanks Dr. Willis for sharing the ocean heat storage data, Dr. Susskind for providing the HIRS Pathfinder OLR dataset, and Dr. Lin Chambers (NASA LaRC) for editorial support. The ERBE/ERBS Nonscanner WFOV and the CERES/Terra Scanner data are provided by the NASA Langley Atmospheric Sciences Data Center in Hampton, Virginia. These data are also available directly on-line from their web site at eosweb.larc.nasa.gov. The ISCCP FD data set is obtained directly on-line from the ISCCP web site at isccp.giss.nasa.gov. The AVHRR Pathfinder ERB data set is obtained on-line from the NOAA web site at www.saa.noaa.gov. The NASA Science Mission Directorate through the CERES project at Langley Research Center funded this study. 


\section{REFERENCE}

Barkstrom, B. R., 1984: The Earth Radiation Budget Experiment (ERBE). Bull. Amer. Meteor. Soc., 65, 1170-1185.

Bess, T. D., G. L. Smith, and T. P. Charlock, 1989: A Ten-year Monthly Data Set of Outgoing Longwave Radiation from Nimbus-6 and Nimbus-7 Satellite. Bull. Amer. Meteor. Soc., 70, 480-489.

Duvel, J.-Ph., M. Viollier, P. Raberanto, R. Kandel, M. Haeffelin, L. A. Pakhomov, V. A. Golovko, J. Mueller, R. Stuhlmann, and the International ScaRaB Scientific Working Group, 2001: The ScaRaB-Resurs Earth Radiation Budget Dataset and First Results. Bull. Amer. Metero. Soc., 82, 1397-1408.

Hansen, J., L. Nazarenko, R. Ruedy, M. Sato, J. Willis, A. Del Genio, D. Koch, A. Lacis, K. Lo, S. Menon, T. Novakov, J. Perlwitz, G. Russell, G. A. Schmidt, and N. Tausnev, 2005: Earth's Energy Imbalance: Confirmation and Implications. Science, 308, 1431-1435.

IPCC, 2001: Climate Change 2001: The Scientific Basis, Cambridge University Press, $870 \mathrm{pp}$.

Jacobowitz, H., L. L. Stowe, G. Ohring, A. Heidinger, K. Knapp, and N. R. Nalli, 2003: The Advanced Very High Resolution Radiometer Pathfinder Atmosphere (PATMOS) Climate Dataset: A Resource for Climate Research, Bull. Amer. Meteor. Soc., 84, 785-793.

Kandel, R., M. Viollier, P. Raberanto, J.-Ph. Duvel, L. A. Pakhomov, V. A. Golovko, A. P. Trishchemko, J. Muller, E. Raschke, R. Stuhlmann, and the International ScaRaB 
Scientific Working Group, 1998: The ScaRaB Earth Radiation Budget Dataset. Bull. Amer. Meteor. Soc., 79, 765-783.

Lee, R. B. III, J. Paden, D. K. Pandey, R. S. Wilson, K. A. Bush, and G. L. Smith, 2002: On-obrit radiometric calibrations of the ERBE active-cavity radiometers on the Earth Radiation Budget Satellite (ERBS): 1984-2002. Proceedings of SPIE, 4814, 7-10 July 2002, Seattle, Washington, USA, 369-379.

Lee, R. B. III, G. L. Smith, K. A. Bush, J. Paden, D. K. Pandey, R. S. Wilson, K. J. Priestley, 2003: On-obrit calibrations of the ERBE active-cavity radiometers on the Earth Radiation Budget Satellite (ERBS): 1984-2002. Proceedings of SPIE, 5234, 8-10 September 2003, Barcelona, Spain, 433-444.

Levitus, S., J. I. Antonov, J. Wang, T. L. Delworth, K. W. Dixon, A. J. Broccoli, 2001: Anthropogenic Warming of Earth’s Climate System. Science, 292, 267-270.

Lin, B, T. Wong, B. A. Wielicki and Y. Hu. 2004: Examination of the Decadal Tropical Mean ERBS Nonscanner Radiation Data for the Iris Hypothesis. Journal of Climate: Vol. 17, No. 6, pp. 1239-1246.

Lindzen, R.S., M.-D. Chou, A. Y. Hou, 2001: Does the Earth Have an Adaptive Iris? Bull. Amer. Met. Soc., 82, 417-432.

Mehta, A., J. Susskind, 1999: Outgoing Longwave Radiation from the TOVS Pathfinder Path A Data Set. J. Geophys. Res., 104, D10, 12,193-12,212.

Stowe, L. L., H. Jacobowitz, G. Ohring, K. R. Knapp, N. R. Nalli, 2002: The Advanced Very High Resolution Radiometer (AVHRR) Pathfinder Atmosphere (PATMOS) Climate Dataset: Initial Analyses and Evaluations. J. Climate, 15, 1243-1260. 
Wielicki, B. A., B. R. Barkstrom, E. F. Harrison, R. B. Lee, III, G. L. Smith, and J. E. Cooper, 1996: Clouds and the Earth's Radiant Energy System (CERES): An Earth Observing System experiment. Bull. Amer. Meteor. Soc., 77, 853-868.

-----, T. Wong, R. P. Allan, A. Slingo, J. T. Kiehl, B. J. Soden, C. T. Gordon, A. J. Miller, S.-K. Yang, D. Randall, F. Robertson, J. Susskind, and H. Jacobowitz, 2002a: Evidence for Large Decadal Variability in the Tropical Mean Radiative Energy Budget. Science, 295, 841-844.

-----, A. D. Del Genio, T. Wong, J. Chen, B. E. Carlson, R. P. Allan, F. Robertson, H. Jacobowitz, A. Slingo, D. Randall, J. T. Kiehl, B. J. Soden, C. T. Gordon, A. J. Miller, S.-K. Yang, and J. Susskind, 2002b: Response: Changes in Tropical Clouds and Radiation. Science, 296, 2095a.

Willis, J., D. Roemmich, B. Cornuelle, 2005: Interannual Variability in Upper-Ocean Heat Content, Temperature, and Thermosteric Expansion on Global Scales. J. Geophys. Res., In Press.

Zhang, Y., W. B. Rossow, A. A. Lacis, V. Oinas, M. I. Mishchenko, 2004: Calculation of radiative fluxes from the surface to top of atmosphere based on ISCCP and other global data sets: Refinements of the radiative transfer model and the input data. $J$. Geophys. Res., 109, D19105, doi:10.1029/2003JD004457, 27pp. 


\section{LIST OF FIGURES}

Figure 1. Time series of ERBS altitude (km) from 1985 to 1999.

Figure 2. Time series of ERBS Nonscanner WFOV deseasonalized tropical mean (20N to 20S) broadband radiation budget anomalies (longwave, shortwave, and net) from 1985 to 1999 with (solid colored lines, Edition3 data) and without satellite altitude correction (black dotted lines, Edition2 data). Anomalies are defined with respect to the 1985 to 1989 period

Figure 3. Time series of ERBS Nonscanner WFOV Edition3 tropical mean (20N to 20S) daytime longwave flux (top, red curve), nighttime longwave flux (top, blue curve), and day-minus-night longwave flux differences (bottom, green curve) from 1985 to 1999.

Figure 4. Time series of ERBS Nonscanner MFOV tropical mean (20N to 20S) daytime longwave flux (top, red curve), nighttime longwave flux (top, blue curve), and dayminus-night longwave flux differences (bottom, green curve) from 1985 to 1999.

Figure 5. Comparison of time series of ERBS Nonscanner WFOV Edtion3_Rev1 deseasonalized tropical mean (20N to $20 \mathrm{~S}$ ) broadband radiation budget anomalies of longwave (top, red curve), shortwave (middle, blue curve), and net (bottom, green curve) radiation from 1985 to 1999 with both satellite altitude correction and shortwave sensor drift correction against the same time series from climate models (black lines are the models mean and the shaded areas are the spread of the minimum and maximum values among models). The climate models are the same models as in Wielicki et al. (2002a). 
Figure 6. Time series of deseasonalized tropical mean (20N to $20 \mathrm{~S})$ broadband radiation budget anomalies (longwave, shortwave, and net) from 1985 to 1999 from the ERBS Nonscanner WFOV Edition3_Rev1 (red), ISCCP FD (blue), HIRS Pathfinder OLR (pink), and AVHRR Pathfinder ERB (green) data records. Anomalies are defined with respect to the 1985 to 1989 period.

Figure 7. Time series of net flux anomalies from 1993 to 2002 from ERBE/ERBS Nonscanner WFOV Edition3_Rev1 (red), CERES/Terra FM1 Scanner ES4 Edition2_Rev1 (green), and ocean heat storage data (blue).

Figure 8 . Time series of deseasonalized tropical mean (20N to $20 \mathrm{~S})$ longwave anomaly (with respect to 1985 to 1989 climatology) between 1979 and 2001 based on the new ERBS Nonscanner WFOV Edition3_Rev1 (Red solid line), Nimbus7 Nonscanner (green dash line) ERBS Scanner (blue solid line), CERES/Terra FM1 Scanner ES4 Edition2_Rev1 (blue dash line), CERES/TRMM Scanner Edition2 (blue circle), ScaRaB/Meteor Scanner (green triangle), and ScaRaB/Resurs Scanner (green circle) dataset. Anomalies are defined with respect to the 1985 to 1989 period. 


\section{LIST OF TABLES}

Table 1. TOA radiative flux changes from the 1980 s to 1990 s from different datasets. Values are given as tropical mean (20N to 20S) for the $1994-1997$ period minus the 1985-1989 period. Dashes are shown where no data is available.

\begin{tabular}{|c|c|c|c|}
\hline Data Source & TOA LW & TOA SW & TOA Net \\
\hline ERBS WFOV Edition 2 & 3.1 & -2.4 & -0.7 \\
\hline ERBS WFOV Edition3 & 1.6 & -3.0 & 1.4 \\
\hline ERBS WFOV Edition3_Rev1 ${ }^{1}$ & 0.7 & -2.1 & 1.4 \\
\hline HIRS Pathfinder & 0.2 & - & - \\
\hline AVHRR Pathfinder ${ }^{2}$ & -1.4 & 0.7 & 0.7 \\
\hline ISCCP FD & 0.5 & -2.4 & 1.8 \\
\hline
\end{tabular}

${ }^{1}$ ERBS WFOV Edition3 with additional user-applied SW sensor drift adjustment

${ }^{2}$ Original uncorrected AVHRR Pathfinder data 


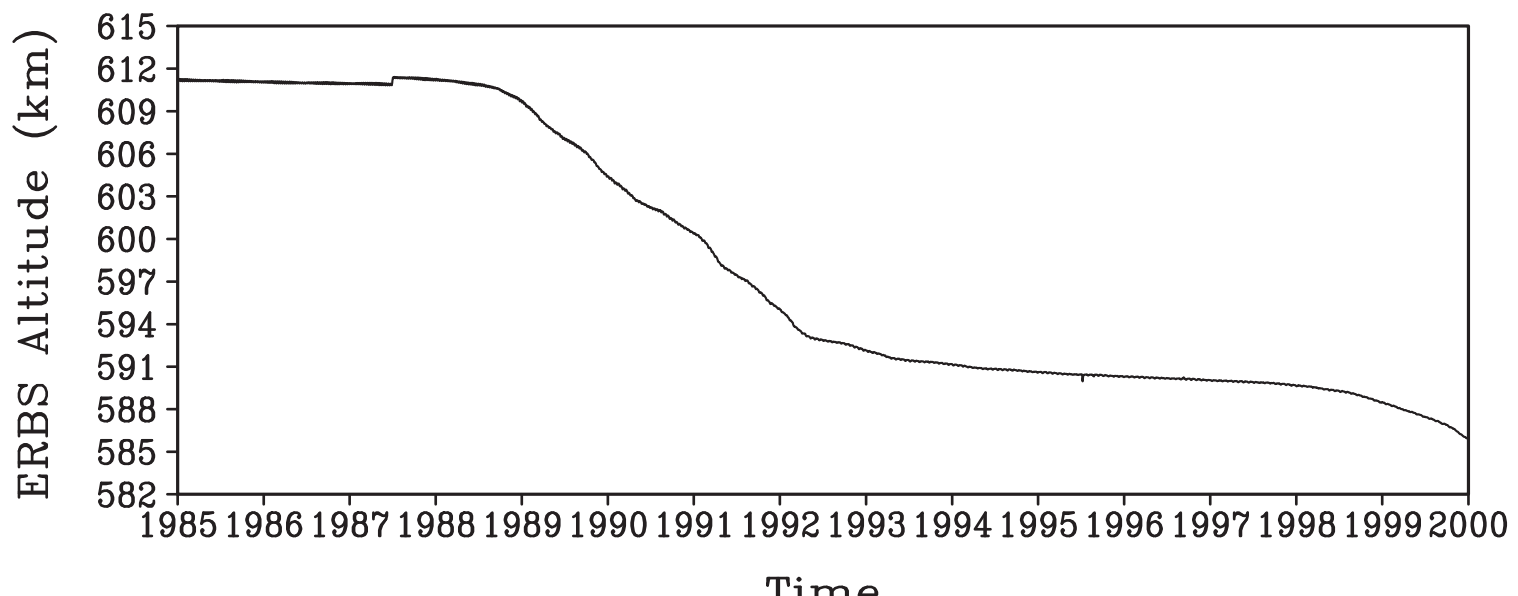

Figure 1. Time series of ERBS altitude (km) from 1985 to 1999. 


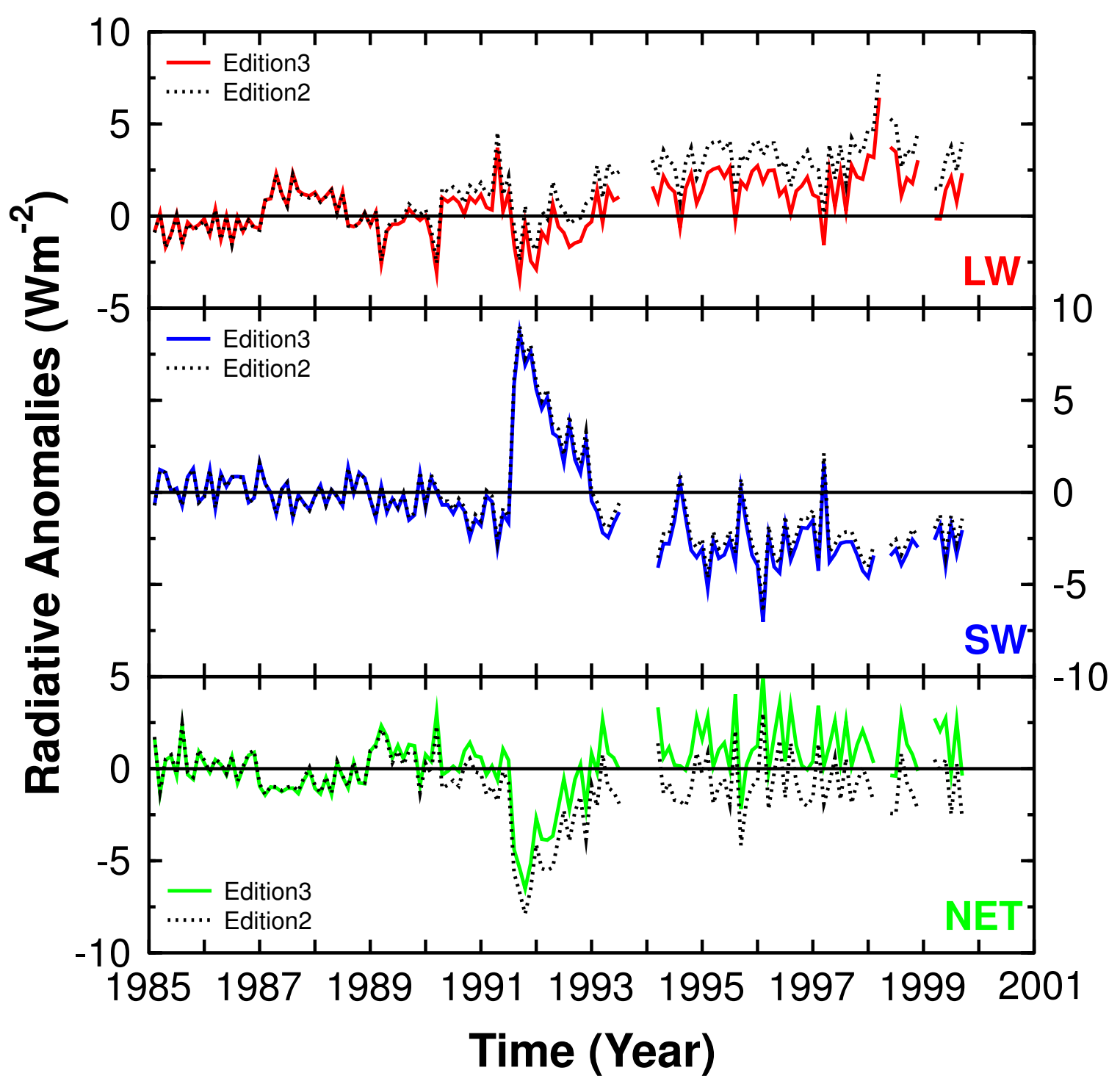

Figure 2. Time series of ERBS Nonscanner WFOV deseasonalized tropical mean (20N to 20S) broadband radiation budget anomalies (longwave, shortwave, and net) from 1985 to 1999 with (solid colored lines, Edition3 data) and without satellite altitude correction (black dotted lines, Edition2 data). Anomalies are defined with respected to the 1985 to1989 period. 


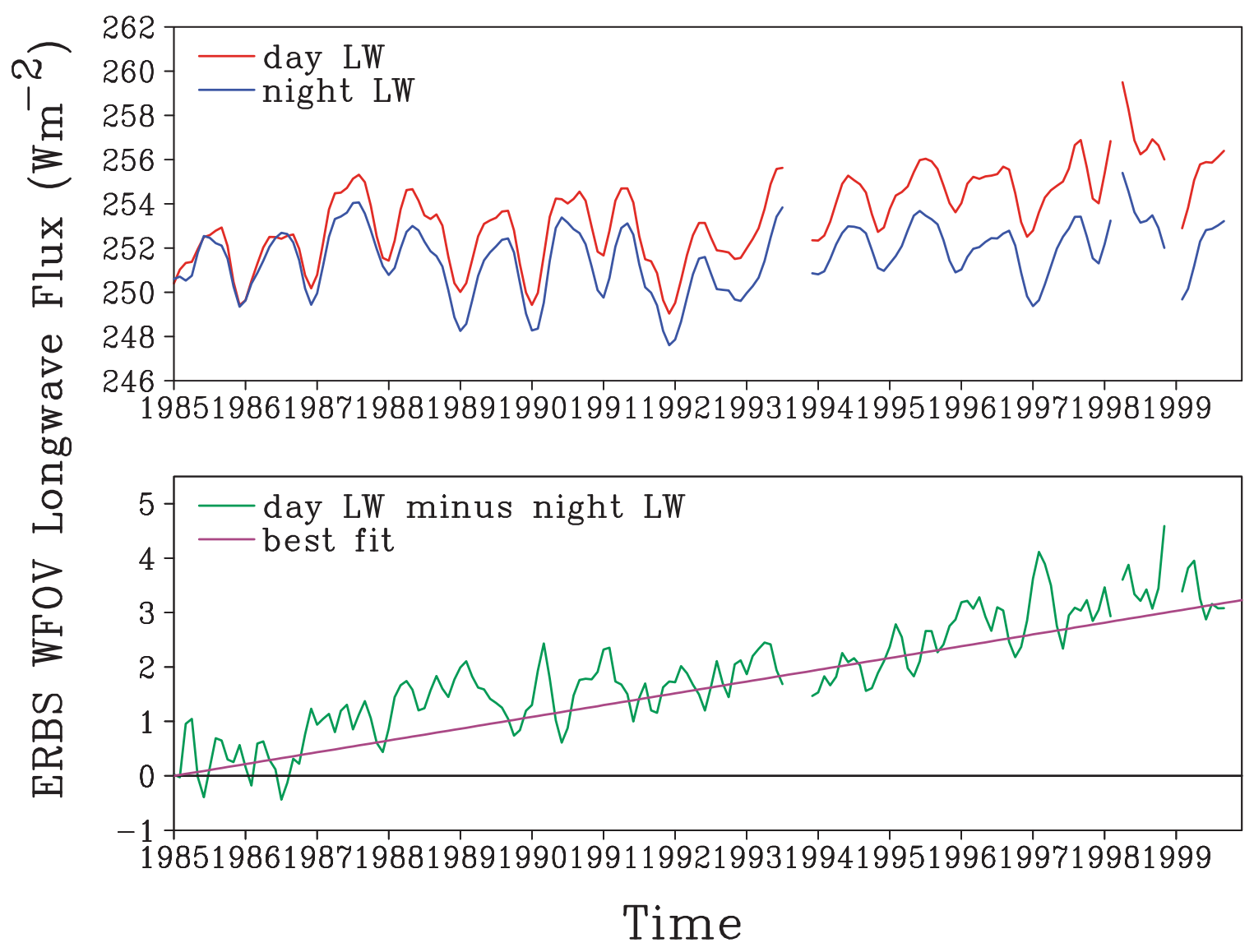

Figure 3. Time series of ERBS Nonscanner WFOV Edition3 tropical mean (20N to 20S) daytime longwave flux (top, red curve), nighttime longwave flux (top, blue curve), and day-minus-night longwave flux differences (bottom, green curve) from 1985 to 1999. 


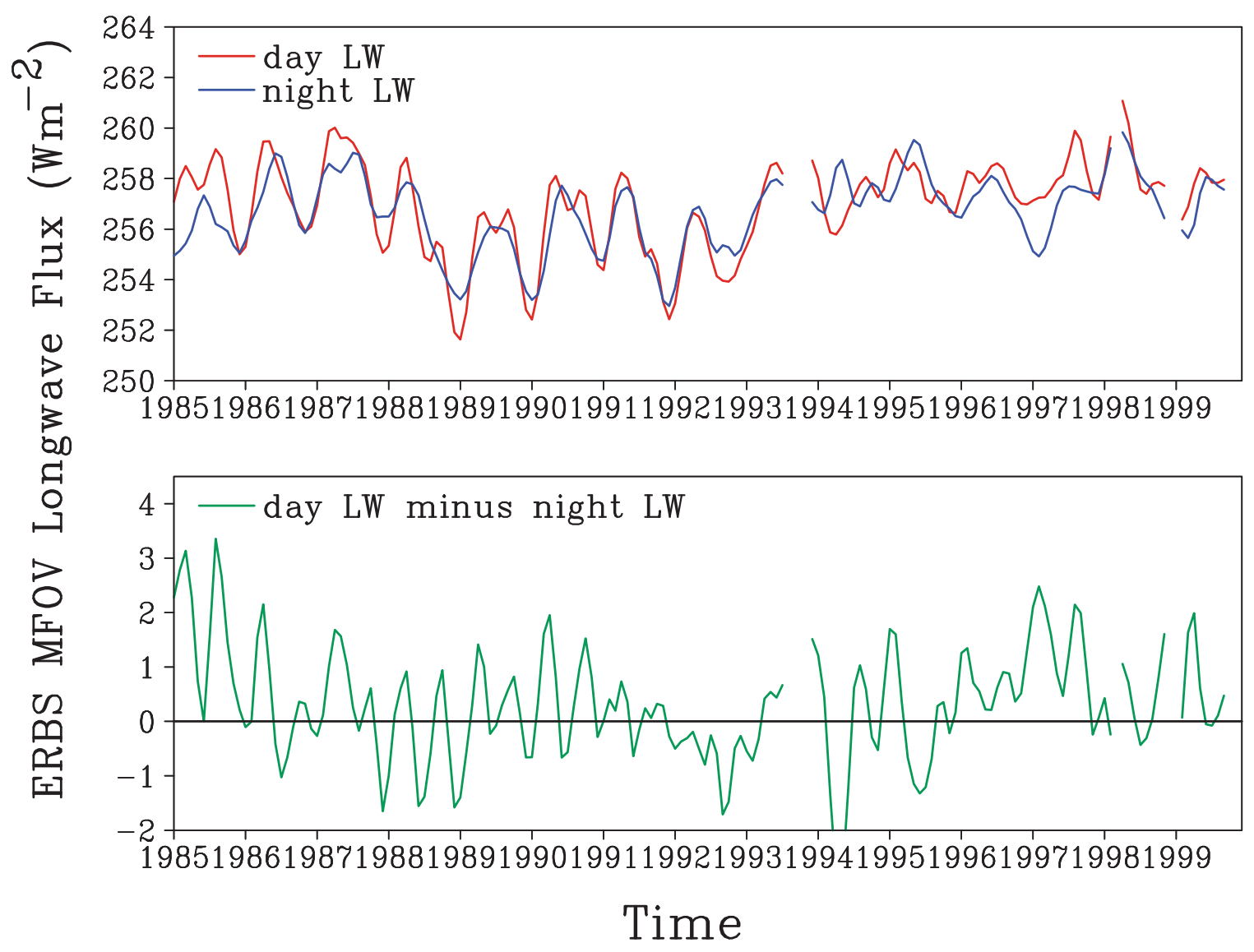

Figure 4. Time series of ERBS Nonscanner MFOV tropical mean (20N to 20S) daytime longwave flux (top, red curve), nighttime longwave flux (top, blue curve), and dayminus-night longwave flux differences (bottom, green curve) from 1985 to 1999. 


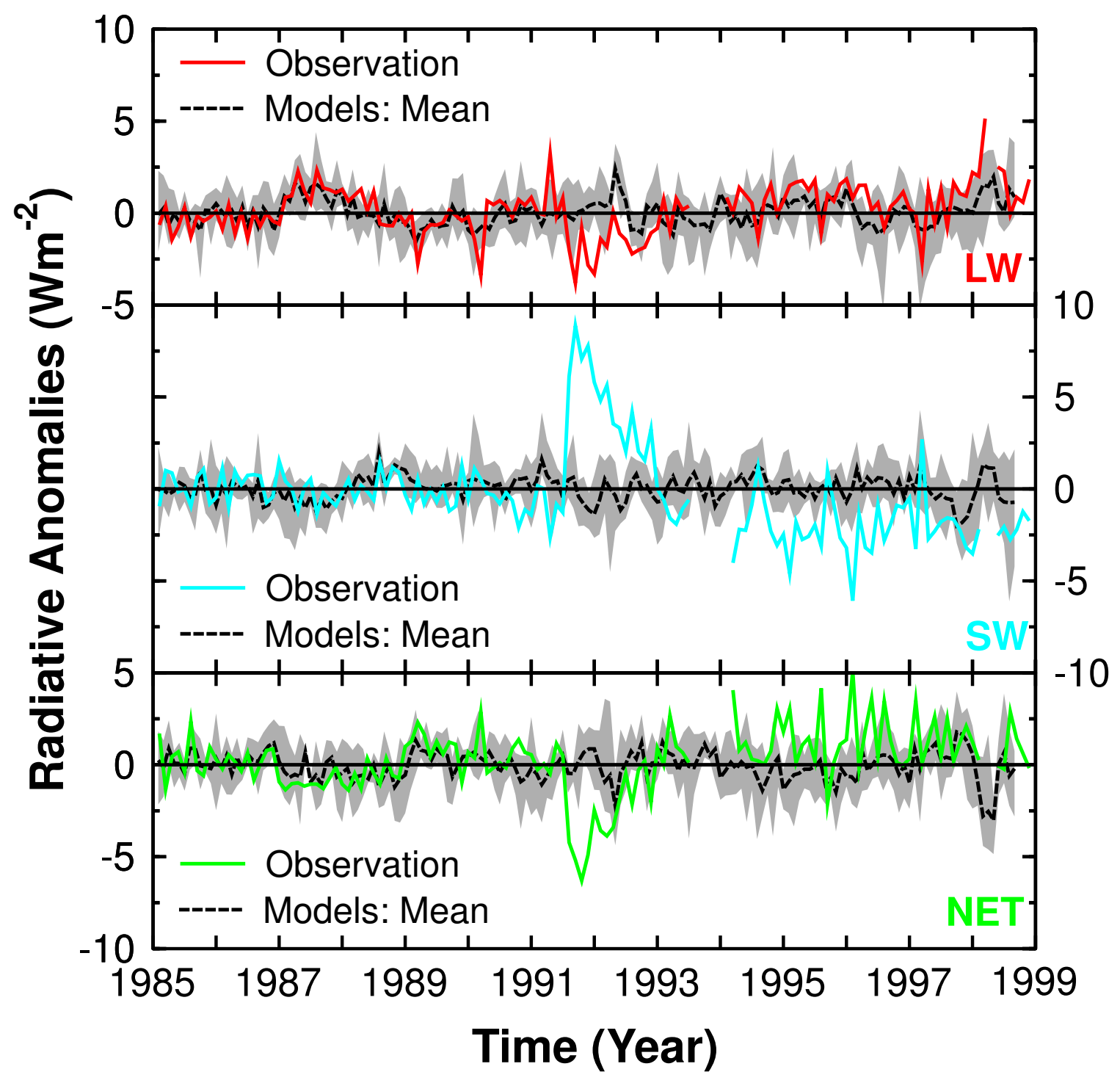

Figure 5. Comparison of time series of ERBS Nonscanner WFOV Edition3_Rev1 deseasonalized tropical mean (20N to $20 \mathrm{~S}$ ) broadband radiation budget anomalies of longwave (top, red curve), shortwave (middle, blue curve), and net (bottom, green curve) radiation from 1985 to 1999 with both satellite altitude correction and shortwave sensor drift correction against the same time series from climate models (black lines are the models mean and the shaded areas are the spread of the minimum and maximum values among models). The climate models are the same models as in Wielicki et al. (2002a). 


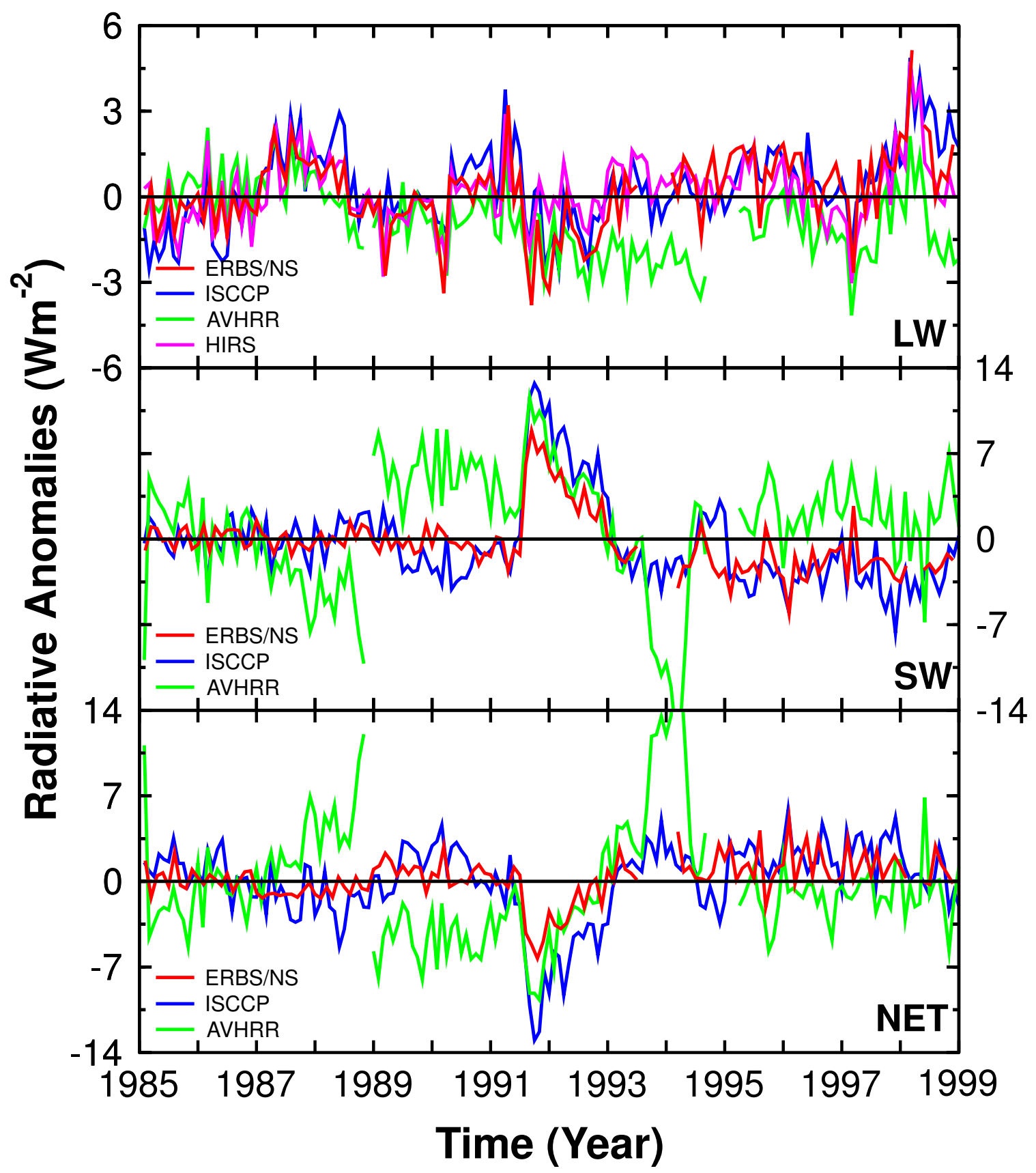

Figure 6. Time series of deseasonalized tropical mean (20N to $20 \mathrm{~S})$ broadband radiation budget anomalies (longwave, shortwave, and net) from 1985 to 1999 from the ERBS Nonscanner WFOV Edition3_Rev1 (red), ISCCP FD (blue), HIRS Pathfinder OLR (pink), and AVHRR Pathfinder ERB (green) data records. Anomalies are defined with respect to the 1985 to 1989 period. 


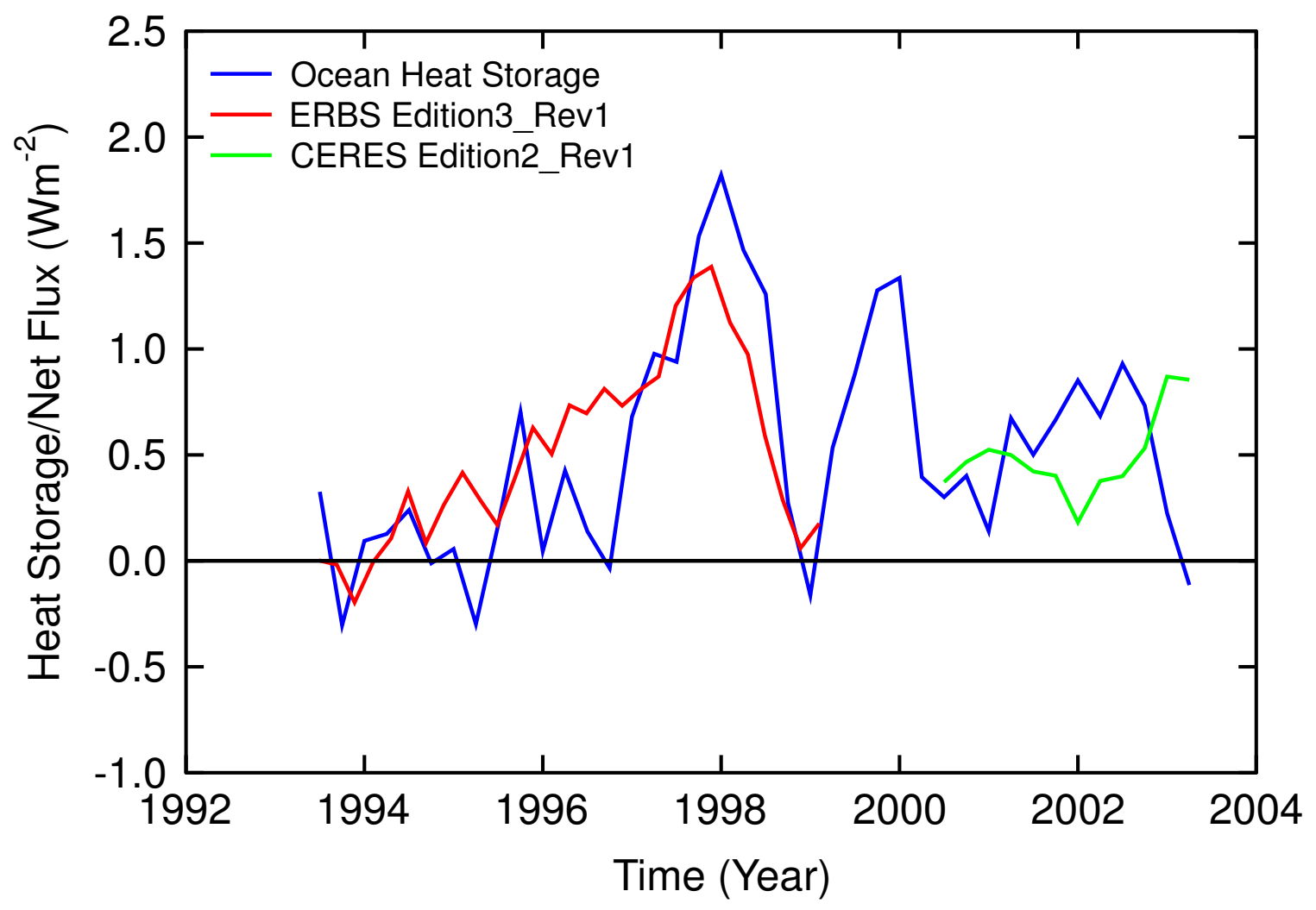

Figure 7. Time series of net flux anomalies from 1993 to 2002 from ERBE/ERBS

Nonscanner WFOV Edition3_Rev1 (red), CERES/Terra FM1 Scanner ES4

Edition2_Rev1 (green), and ocean heat storage data (blue). 


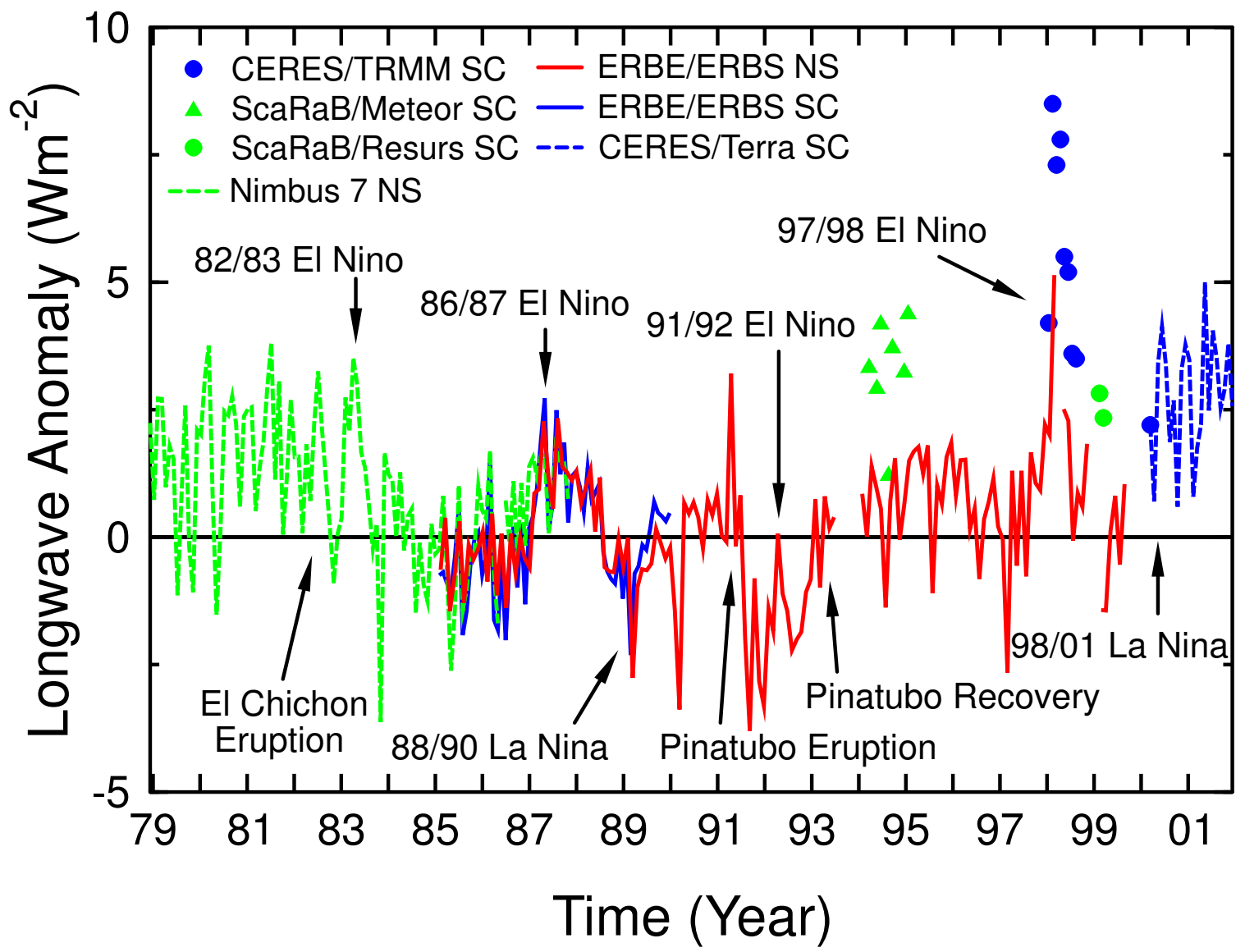

Figure 8 . Time series of deseasonalized tropical mean ( $20 \mathrm{~N}$ to $20 \mathrm{~S})$ longwave anomaly (with respect to 1985 to 1989 climatology) between 1979 and 2001 based on the new ERBS Nonscanner WFOV Edition3_Rev1 (Red solid line), Nimbus7 Nonscanner (green dash line), ERBS Scanner (blue solid line), CERES/Terra FM1 Scanner ES4 Edition2_Rev1 (blue dash line), CERES/TRMM Scanner Edition2 (blue circle), ScaRaB/Meteor Scanner (green triangle), and ScaRaB/Resurs Scanner (green circle) dataset. Anomalies are defined with respect to the 1985 to 1989 period. 